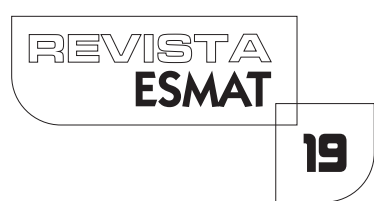

\title{
CRIME CONTROL OU DOING BUSINESS : QUELLE POLITIQUE PENALE EN MATIERE ECONOMIQUE ET FINANCIERE? ${ }^{1}$
}

CRIME CONTROL OR DOING BUSINESS: WHAT CRIMINAL POLICY IN ECONOMIC AND FINANCIAL MATTER?

Léonid Golovko

Professeur à la Faculté de Droit de l'Université d'Etat de Moscou (Lomonossov), Directeur du Centre de Procédure pénale et de Justice.

\section{RESUME}

Comment expliquer certaines tentatives de prévoir des dispositions pénales déragatoires en matière économique et financière, notamment en procédure pénale ? ॥ est possible de dégager deux logiques hypothétiques, celle duCrime Control, où le droit pénal doit devenir plus efficace dans la lutte contre la criminalité économique et financière, et celle duDoing Business, où le droit pénal doit quasiment disparaître du champs économique pour ne pas nuire à l'activité commerciale. Le droit pénal russe se présenteactuellement, plutôt comme un exemple du mouvement Doing Business, ce qui apporte plus de problèmes que de résultats positifs. II en résulte que la logique Doing Business ne peut être un principe de politique pénale en matière économique et financière.

MOTS-CLES: Politique pénale; procédure pénale; matière économique et financière; Russie.

'Cet article est basé sur le rapport fait par l'auteur lors des Journées internationales 20 I 5 de la Société de législation comparée, qui ont eu lieu à Paris le 8 et 9 avril 20 I 5, dans le cadre de l'atelier «Les procédures répressives contre la grande délinquance économique et financière». La première version du texte a été mise en ligne sur le site comitasgentium.com. En tant que tel, l'article n'a jamais été publié avant. 


\begin{abstract}
How to explain some efforts to create a special criminal law's provisions in economic and financial matter, in particular in criminal procedure? It is possible to reveal two hypothetical logics: the logic of Crime Control, according to which the criminal law has to become more efficient for fighting the economic and financial criminality, and the logic of Doing Business, according to which the criminal law almost has to disappear from the economic sphere not to disturb the economic activity. The Russian criminal law is actually an example of the Doing Business criminal policy's movement, which brings more problems than positive results. It follows that the Doing Business logic cannot be a criminal policy's principle in economic and financial matter.
\end{abstract}

KEYWORDS: Criminal policy; criminal procedure;economic and financial matter; Russia.

\title{
I INTRODUCTION
}

La dernière décénnie, le droit pénal et la procédure pénale russes sont institutionnellement très favorables à tous ceux qui peuvent être considérés comme entrant dans la catégorie des entrepreneurs, c'est-à-dire des acteurs du secteur privé des affaires. Le nombre des dispositions spéciales créant un régime pénal particulier (dérogatoire) à leur égard augmente et cette tendance est loin de s'essouffler. Le prétexte politico-économique est connu : favoriser le développement économique, attirer les investissements etc.

Même si, techniquement, il est possible qu'il existe sur ce plan certaines spécificités russes, conceptuellement cette démarche politico-législative nationale s'inscrit parfaitement dans le contexte général.Les relations entre le pénal et l'économie deviennent de plus en plus tendues, «à partir des années 1990, les théories néolibérales, prônant l'extension du marché à l'ensemble du corps social, ont facilité l'intégration de doctrines adaptant le management aux institutions publiques» (D'HERVÉ, p. 49-50). II est donc théoriquement admissible de traiter le cas russe, non comme un phénomène totalement isolé, mais plutôt comme l'expérimentation d'un législateur, qui s'est avancé un peu plus loindans le maillage de tendances globales. De ce fait, il est également possible d'essayer de dégager de l'exemple russe quelques réflexions, qui dépassent le cadre du droit national et peuvent être conceptualisées à un niveau moins technique, plus théorique.

En ce sens, lorsque l'on aborde le problème de l'existenceen Russie de spécificitéspénales à caractère dérogatoire en matière économique et financière ${ }^{2}$,surtout de la procédure pénale (en droit pénal de fond une telle spécificité est

\footnotetext{
${ }^{2}$ Il convient de rappeler deux approches concernant la délinquance économique et financière :
} 
évidente), une simple analyse du droit positif ne semble pas être suffisante. Le plus important est de comprendre la logique politico-juridique de ces spécificités (ou dérogations) qui peuvent apriori s'inscrire dans deuxmouvements hypothétiques et alternatifs de politique pénale en matière économique et financière qu'il est possible de dégager:

a) le mouvement du Crime Control, qui est très bien conceptualisé (PACKER, p. I), dans le cadre duquel les procédures dérogatoires se présententcomme un instrument ayant pour but de renforcer la lutte contre la délinquance économique et financière (il s'agit, pour ainsi dire, de la logique des pénalistes);

b) le mouvement du Doing Business, qui est très peu, voire pas du tout, conceptualisé doctrinalement en matière pénale, pour lequel les procédures dérogatoires serventd'un instrument pour libéraliser le secteur économique et minimiser le risque pénal à l'encontre des acteurs de l'activité économique (il s'agit, pour ainsi dire, de la logique des économistes).

Si en droit pénal de fond les tendances sont moins claires, voire plutôt contradictoires, puisque l'on voit, d'une part, se mettre en oeuvre des mécanismes de dépénalisation et de décriminalisation de certaines infractions pénales économiques et financières et, d'autre part, l'apparition de nouvelles incriminations ${ }^{3}$, en procédure pénale toutes les dérogations en matière économique s'inscrivent dans la logique du Doing Business. C'est-à-dire que le but du législateur, poussé il est vrai par le discours économistenéolibéral sur le plan abstrait et un lobby des grands mileux d'affaires assez puissant sur le plan concret, est de créer en droit pénal économique un régime procédural plus favorable qu'en matière du droit pénal ordinaire. Cette tendance s'est manifestée en Russie sous la présidence de D. Medvedev, surtout entre 2009 - 20 I I, pour se ralentir un peu avec le retour au sommet du pouvoir de V. Poutine en 20 I 2, sans

celle criminologique et celle institutionnelle. Si sur le plan criminologique la fameuse notion de White Collar Criminality comprend à la fois toute sorte de comportements relatif au «business» (par exemple, SUTHERLAND, p. I), peu importe qu'il s'agisse de la délinquance des fonctionnaires ou de la délinquance des particuliers en matière économique (fraude fiscale, corruption, manipulation des cours etc., cf. par exemple, IBID, p. 2-3), sur le plan institutionnel le droit pénal instaure une nette distinction entre les infractions contre la fonction publique (corruption, abus de pouvoir etc.) et les infractions en matière économique et financière stricto sensu, c'est-à-dire contre les biens, en matière fiscale, relatives au fonctionnement de l'entreprise etc. Sans sous-estimer l'importance de la démarche criminologique, les tendances et les spécificités, que l'on analyse ici, ne concernent que la matière économique et financière stricto sensu,et non pas le phénomène des infractions contre la fonction publique, pour lequel la logique qui nous intéresse ne s'applique pas.

${ }^{3}$ Sur ces réformes en matière de droit pénal de fond voir notre article : GOLOVKO, Léonid. Panorama de droit pénal russe (2008 - 20I I), Revue pénitentiaire et de droit pénal, 20 I I, 4. P. $866-867$. 
être pourtant complètement abandonnée ou mise en doute. Sur le plan formel, il ne s'agit pas d'un chapitre spécial dans le Code de procédure pénale (CPP) russe de 2002, même si une telle proposition apparaît de tempsen temps parmi les initiatives lancées par leCommissioner for Entrepreneurs' Rights russe (médiateur en charge de la protection des droits des entrepreneurs auprès du Président russe) ${ }^{4}$, mais plutôt de différentes dispositions dispersées dans le texte du CPP, c'est-à-dire non systématisées.

La première question qui se pose, alors, est de comprendre pourquoi un tel choixa été fait par le législateur russe, qui avance de son côté une justification institutionnelle (2). D'autant plus que ce choix de politique pénale en matière économique et financière a directement et, je dirais même, objectivement, conduit à des critiques juridiques difficilement surmontables (3).

\section{LAJUSTIFICATIONDU CHOIX}

La volonté de rejeter totalement la logique du Crime Control en matière économique et financière a nécéssité des recherches sur la conceptualisation de cette démarche (2. I).Mais sans même attendrele résultat de cette conceptualisation, le législateur russe s'est déjà très concrètement lancédans le processus de consécration de la logique du Doing Business par une série de réformes de la procédure pénale (2.2).

\section{I Le rejet du Crime Control}

Comment justifier, dans une société moderne comme la nôtre, que la législation pénale soit plus favorable, tant au niveau normatif, qu'au niveau de sa mise en oeuvre, à un homme d'affaires, qu'à une autre personne, qui aurait commisune infraction en déhors du domaine du «business»? La tâche intellectuelle ne semble pas être facile.

Les débats doctrinaux russes ont démontré qu'il existe deux types de réponse, essayant de justifier cette différenciation de la réaction pénale ratione materiae (c'est-àdire en matière économique et en matière non économique) : une,intellectuellement plus ambitieuse qui tente de mettre en cause la notion même du droit pénal des affaires, et l'autrethéoriquement plus modérée, qui se restreint à la démonstration du visage non-marginal du «businessman» par rapport aux «vrais» criminels.

Si l'on commence par l'approche modérée des tentatives de justification, il convient de noter que le droit pénal russe est construit autour de la notion de danger social, qui sert de critère à la pénalisation den'importe quel comportement(art. 14 du Code pénale (CP) russe de 1997). Donc, pour justifier que le droit pénal, notamment dans sa branche

${ }^{4}$ Cette fonction a été crée en juin 2012 et joue un rôle fondamental pour tout ce qui concerne la libéralisation du droit pénal. 
procédurale, doive être plus favorable en matière économique et financière, et sans contester pour autant la légitimité de la sanction pénale en tant que telle, il est nécéssaire de prouver que le danger social, provoqué par une infraction en cette matière est moins grave que le danger social provoqué par d'autres infractions pénales « non économiques ». Est-ce théoriquement réaliste?

A vrai dire, vue l'impossibilité évidente de démontrer qu'un dommage matériel causé par un simple vol a priori dépasse le dommage causé par une activité criminelle en matière économique et financière, ce qui est tout simplement absurde, ce discours nous amène à la logique purement anthropologique du phénomène pénal, où la notion de danger social se personnalise, se transforme en un «criminel physiquement dangereuX», réduisant le champ pénal presqu'aux seuls actes violents. En quelques sorte, il s'agit d'un retour à l'époque d'avant E.-H. Sutherland, puisque cette démarche met en doute la conception même de White Collar Criminality, en tout cas hors champs des infractions pénales contre la fonction publique, c'est-à-dire commises par les fonctionnaires d'Etat. En d'autres termes, l'archaïsation du discours pénal, construit à nouveau uniquement autour du criminel physiquement dangereux et des actes violents, se présente comme un obstacle théoriquement difficilement surmontable pour justifier le rejet,sur la base d'un danger social soi-disant moins important, de la logique du Crime Control en matière économique et financière.

II a donc fallu lancer un projet intellectuel plus ambitieux pour tenter de prouver que le fonctionnement de l'économie moderne ne supporte pas, par principe, la pression hypothétique de la sanction pénale et de la procédure pénale, ce qui doit provoquer le basculement radical des rapports classiques entre le droit pénal et le droit privé, en «privatisant» le pénal et ainsi libérant l'entrepreneur (homme d'affaires) presque de tout risque pénal, en tout cas dans les limites de son activité professionnelle.

La culmination de cette réflexion a eu lieu dans le cadre de trois tables rondes, organisées par un groupe d'initiative russe, transformé bientôt en Centre de recherches juridiques et économique, avec le soutien financier et organisationnel du McGill University, qui se sont tenues à Moscou le 27 novembre 2008, le 2 mars 2009 et le 5 juin 2009 avec la participation de différents experts (juristes, économistes etc.) russes et étrangers. Le bilan a été présenté en automne 2009 sous la forme d'une «monographie collective internationale», gros volume de 600 pages, composé de quelques articles et surtout des sténogrammes complets des trois tables rondes, dont le compte rendu synthétique a été, par ailleurs, traduit en anglais comme annexe à la monographie(«Rule of Law in Russia - Issues of Implementation, Enforcement and Practice») ${ }^{5}$ Un an plus tard, la même équipe, mais cette fois-ci avec le soutien non seulement du McGill University, mais également du très influent, à l'époque de la présidence de D. Medvedev, Institut du développement moderne (INSOR), de la non moins influente École des Hautes Études en Sciences Économique et du Liberal Mission Foundation (un think thank des économistes russes), publie un ouvrage intitulé «La Conception de la modernisation de la 
législation pénale russe en matière économique» ${ }^{5}$, dont la présentation a été organisée directement à la Douma (Chambre basse du Parlement russe).

Est-ce que les ouvrages mentionnés, lorsque l'on les analyse sur le fond, contiennent cette «pierre philosophale», cette formule magique théorique qui permette de prouver l'incompatibilité totale des mesures pénales classiques avec l'économie moderne, voire au moins de protéger un entrepreneur de toute possibilité de poursuite pénale dans le cadre de son activité professionnelle? II ne semble pas.Sur le plan théorique, on ne peuty trouver que le slogan sur la «criminalisation artificielle de l'activité économique» et le fameux Argumentum ad Investitiam, qui, dans ce contexte, est concrétisé par l'idée que le droit pénal national apparaît comme un obstacle majeur pour attirer les investissements dans le pays. Rien de plus.

Néanmoins, malgré l'échec évident pourréellement conceptualiser la démarche, le législateur a préféré ne pas attendre et a rejeté la logique du Crime Control en matière économique et financière pour se lancer sur le chemin alternatif du Doing Business. C'est à ce moment que le droit pénale russe a connu plusieures réformes, notamment en procédure, prévoyant la création de régimes dérogatoires en matière économique et financière. Pour être exact, certaines de ces réformes réflètent des débats beaucoup plus concretslancés par les instigateurs des tables rondes de 2008 - 2009.

\section{2 La consécration du Doing Business}

Ce mouvement législatif peut être illustré à travers trois exemples concernant les modifications apportées au CPP russe.

I) Tout d'abord, il s'agit d'une série de lois visant à minimiser, voire à exclure complètement, l'utilisation de la détention provisoire en matière économique et financière.

L'adoption de la loi du 29 décembre 2009, interdisant l'application de la détention provisoire à l'égard de toute personne mise en examen pour une infraction fiscale (art. 198, 199, 199.1, 199.2 du CP ${ }^{8}$ ), si elle respecte ses obligations procédurales dans le cadre du contrôle judiciaire, au départ se présente comme une réaction très rapide à la

\footnotetext{
${ }^{5}$ Verkhovenstvo prava i problemy ego obespetchenia $\vee$ pravoprimenitelnoi praktike (mezhdunarodnaia kollektivnaya monografiya). Moscou. Éd. Statut. 2009 (en russe). Le titre de la version anglaise: Rule of Law in Russia - Issues of Implementation, Enforcement and Practice.

${ }^{6}$ Kontseptsiya modernizatsii ugolovnogo zakonodatelstva $\vee$ ekonomitcheskoi sfere. Moscou. 2010 (en russe).

${ }^{7}$ Ibid. P. II.

${ }^{8}$ Voir le texte de ces incriminations par exemple en français, sauf celles qui ont été ajoutées plus tard, dans : Le Code pénal russe de 1997, trad. de L. Golovko, sous la dir. de J. Pradel. Paris: Cujas, 1998.
} 
fameuse affaire Magnitski, très médiatisée, dans laquelle un jeune juriste-fiscaliste, accusé de complicité de fraude fiscale, est décédé en détention provisoire en novembre 2009.

Ensuite la loi du 7 avril 2010 est intervenue pour ouvertement et officiellement supprimer presque totalement l'application de la détentiondans le cadre de l'activité commerciale, sauf bien évidemment dans les cas où la personne concernée ne respecte pas les obligations imposées par le contrôle judiciaire, et sansque cela ne concerne les activités non commerciales. En premier lieu, le législateur a sensiblement allongé la liste, déjà établie par la loi du 29 décembre 2009 pour les infractions fiscales, jusqu'à une grande partie des infractions économiques et financières(art. 17| - 174, 174. I, 176 178, 180 - | 83, | 85 - 185.4, 190 - 199.4 du CP) telles que notamment le blanchiment d'argent, l'activité bancaire illégale (sans autorisation), la restriction de la concurrence etc., où la détention est dès lors complétementinterdite. En second lieu, la même loi du 7 avril 2010 a exclu la détention pour trois infractions de droit commun (art. I59 - 159.3, 159.5, 159.6-différentes types d'escroquerie ; art. 160 - détournement et dilapidation ; art. 165 - fait d'avoir causé un préjudice patrimonial par fraude ou par abus de confiance), lorsqu'elles «sont commis dans le cadre de l'activité commerciale»". Autrement dit, il s'agit d'une restriction de la détention non in rem, mais plutôt in personam, où, par exemple, la même infraction d'escroquerie laisse la possibilité à la détention, si elle est commise dans un cadre quotidien, et exclue la détention, si elle est commise par un homme d'affaires dans le cadre de son activité professionnelle.

2) II convient de citer ensuite une autre réforme importante, qui a introduit par les lois du 29 décembre 2009 et du 7 décembre 20 I I dans la procédure pénale russe une sorte de transaction en matière économique et financière.

En ce qui concerne les infractions fiscales, si la personne poursuivie est d'accord pourrembourser à l'Etat, avant le jugement définitif, toutes les sommes exigées, y compris toutes les amendes prévues par la législation fiscale, la poursuite pénale doit être impérativement arrêtée soit par l'autorité d'instruction, soit par le juge (nouvel article 28. I du CPP russe).

Le même schéma s'applique pour les infractions économiques, même s'il est un peu plus compliqué. II existe une liste d'infractions économiques prévue par le même art. 28. I du CPP ${ }^{10}$ et dont l'élargissement est effectué de temps en temps (notamment un élargissement important a été éffectué par la loi du 27 décembre 20 18), le plus souvent suivant les initiatives du médiateur en charge de la protection des droits des

${ }^{9}$ Ces deux listes ont été à nouveau élargies (notamment pour l'art. 201 du CP - l'abus des pouvoirs par une personne exercant la gestion dans une société commerciale) par les lois du 29 juillet 2017 et du 27 décembre 2018 modifiant l'art. 108 du CPP russe.

${ }^{10}$ Cette liste comporte une trentaine d'infractions économiques (art. 170.2, I71, 171 I I, 172, 176, 177 etc. du CP). 
entrepreneurs auprès du Président russe. Si une personne poursuivie est prête à rembourser les dommages-intérêts causés à l'Etat, à une personne morale ou à un particulier, et de surcroît à payer au budget d'Etat une somme correspondant à 2 fois le montant du dommage causé par l'infraction, la poursuite pénale doit être également impérativement arrêtée.

Néanmoins, lorsque l'on apprécie cette nouvelle procédure russe, il ne s'agit pas, semble-t-il, d'une transaction pénale au sens strict du terme, mais plutôt d'une quasitransaction. En fait, la version russe ne prévoit aucune négociation dans le style américain (plea) entre les autorités répressives et la personne mise en exament pour une infraction économique ou financière. Les autorités répressives ne peuvent qu'informer la personne de ses droits prévus par l'art. 28. I du CPP sans pouvoir négocier la somme à payer (elle est prévue par la loi). Et si la personne remplie les conditions, la poursuite doit être arrêtée d'office. Ce mécanisme législatif ressemble plutôt à une certaine dépénalisation conditionnelle a posteriori.

3) Enfin, le troisième exemple concerne la loi du 6 décembre 2011 , qui a introduit dans le CPP russe une disposition selon laquelle la poursuite pénale pour une fraude fiscale ne peut pas être engagée d'office par les autorités répressives, sans une demande officielle de l'administration fiscale non pénale. C'est-à-dire qu'il revient d'abord à l'administration fiscale d'établirl'infraction pénale hypothétique, pour ensuite être en mesure de s'adresser aux autorités répressives afin de déclencher la poursuite pénale et de procéder à l'instruction de l'affaire, sinon la poursuite pénale ne peut pas avoir lieu.

Le paradoxe de cette réforme réside dans l'absence de pouvoirs de police judiciaire " de l'administration fiscale, qui n'a donc normalement aucun instrument pour découvrir la fraude, sauf si tous les éléments nécéssaires ne sont pas complaisamment réunis et présentés par le contribuable lui-même. II n'est pas étonnant que cette démarche législative ait quasiment bloqué la lutte en Russie contre les fraudes fiscales et c'est pourquoi il a bientôt fallu revenir sur cette réforme. Finalement, la loi du 22 octobre 2014 a abrogé la disposition introduite en 201 I. Actuellement les autorités répressives peuvent agir d'office en matière de fraudes fiscales mais, en contrepartie, elles sont,dans tous les cas, obligées de demander l'avis écrit de l'administration fiscale, qui est pourtant subordonnée à la libre appréciation des preuves et ne lie donc pas les autorités répressives. Quoi qu'il en soit, la loi du 6 décembre 20 I I, même assouplie par la loi du 22 octobre 2014 , et la polémique qui l'entoure, s'inscrivent parfaitement dans la logique du Doing Business en matière pénale économique et financière.

\footnotetext{
" II est à noter que la police fiscale a été supprimée en Russie en 2003 (sa compétence a été transférée vers les services anti-fraude du Ministère de l'Intérieur). La loi du 6 décembre 20 I I n'a pas même envisagé la restauration de la police fiscale au sein de l'administration fiscale.
} 


\section{LACRITIQUEDU CHOIX}

Le passage du législateur pénal russe à la logique du Doing Business, conjugué à l'impossibilité évidente de juridiquement conceptualiser cette logique, a provoqué une critiqie immédiate tant sur le plan technique (3. I) que politique (3.2).

3. I La critique technique via la dépersonnalisation de l'activité commerciale

En tenant compte du caractère strictement personnel de la responsabilité pénale et, en revanche, du caractère très imprécis de cette «matière économique et financière», la question qui se pose est de savoir vers qui doit être orientée la politique pénale Doing Business, c'est-à-dire qui doit être son bénéficiaire concret sur le plan personnel, individuel? Cette question se complique par le fait que l'idée du Doing Business, d'origine économique et abstraitement très claire, se traduit dificillement en termes juridiques concrets. Comment déterminer juridiquement ce «faiseur du business», vu l'interopérabilité de l'économie moderne? Comment choisir un seul «veau d'or» économique, qui doit légitimement ressentir la complaisancedu droit pénal, s'li y en a plusieurs,vue la structure actuelle de l'économie, où se croisent le producteur, le consommateur, le vendeur ou le créateur, sans pouvoir dégager, concrètement, celui dont l'apport à la richesse économique sera décisif pour qu'il puisse obtenir une protection spéciale contre la loi pénale?

Sans déjà pouvoir, semble-t-il, répondre à ces questions sur le plan conceptuel, le législateur russe s'estréjoui, au départ, de la technique de codification, qui permet de retrouver dans le CP un chapitre spécial, intitulé «Infractions pénales en matière économique». Il est évident que pour chaque incrimination concernée il existe un auteur spécial, ce qui permet d'éviter de se poser la question de l'application de la logique du Doing Businesssur le plan personnel (in personam). De là résulte cette démarche formelle, qui se traduit par le recours à la technique des listes concrètes d'infractions concernées (énumération exhaustive), qui aide à établir le champ d'application des procédures dérogatoires, telles que l'interdiction de la détention provisoire ou la quasitransaction pénale (art. 28. I du CPP).

Cependant, la démarche formelle a immédiatement provoqué deux problèmes. Le premier réside dans le fait que la codification pénale a sa propre logique, en classifiant assez souvent des infractions et les considérant comme «économiques» pour des raisons assez lointaines de celles du Doing Business. Tel est le cas, par exemple, de la corruption des sportifs, des arbitres de sport etc. (art. I 84 du CP) qui a été, à juste titre du point de vue du législateur, classée parmi les infractions en matière économique(il s'agit d'une infraction contre l'économie du sport et les intérêts du consommateurs), mais qui figurait initiallement parmi les infractions prévues par l'art. 28. I du CPP, où il existe un régime dérogatoire, favorable aux auteurs des infractions concernées. Dans cette perspective, le résultat de cette démarche purement formelle peut parfois surprendre, puisqu'il est difficile de supposer que le législateur ait ainsi proclamé safaveur à l'égard du Doing Business en matière de corruption des sportifs et des arbitres de sport. 
Cette incrimination a été retiré relarivement rapidement de la liste prévue par l'art. 28. I , mais l'exemple reste significatif.

Un autre problème semble être encore plus important. La démarche formelle, qui se concentre autour du chapitre spécial du CP relatif aux infractions en matière économique et financière, ne permet pas de résoudre la question des infractions de droit pénal commun, se situant «à la frontière du droit pénal des affaires» (BONFILS, p. 73). II s'agit surtout des infractions contre les biens et en premier lieu de l'escroquerie, «une infraction essentielle en droit pénal des affaires» (IBID, p. 79), et de ses voisins (détournement et dilapidation des biens, abus de confiance etc.). La logique du Doing Business ne peut pas se permettre de les oublier, sans pour autant que la technique de codification ne soit utilisable. Ainsi, les incrimination concernées se retrouvent en déhors du chapitre du CP relatif aux infractions économiques et financières stricto sensu. II a fallu donc passer de la démarche formelle à la démarche matérielle.

En fait, la loi précitée du 7 avril 2010 a exclu au début la détention provisoire pour trois infractions de droit commun (art. 159 - escroquerie ; art. 160 - détournement et dilapidation ; art. 165 - fait d'avoir causé un préjudice patrimonial par fraude ou par abus de confiance), mais uniquement lorsqu'elles «sont commises dans le cadre de l'activité commerciale». Comme nous l'avons déjà précisé, il s'agit de la restriction de la détention non in rem, mais in personam, où, par exemple, la même infraction d'escroquerie laisse la possibilité de la détention, si elle est commise dans sa dimension quotidienne, et exclue la détention, si elle est commise par un homme d'affaires dans le cadre de son activité professionnelle.

Mais de qui s'agit-il concrétement? On n'est plus au XIXème siècle, où le commerce était personnalisé et construit autour de la fameusenotion de «commerçant». Aujourd'hui, dans le contexte d'une économie dépersonnalisée faite de sociétés notamment anonymes, les juges russes se sont retrouvés confrontésà desérieuses difficulés pour élaborer le champ d'application de la loi du 7 avril 2010 in personam. Comment, par exemple, traiter les gérants des entreprises ou autres personnes souvent embauchées par contrat de travail, qui, dans l'économie actuelle, prennent des décisions, c'est-à-dire «font du business», sans avoir le titre formel de commerçant ou autre titre ouvrant droit à ce régime d'exception?La précision de la Cour suprême fédérale,qui a énoncé dans son arrêt d'assemblée plénière du 10 juin 2010 que la notion d'activité commerciale, prévue par l'article 2 du Code civil, s'applique à la loi du 7 avril 2010 sans aucune exception, n'a pas semblée satisfaisante, parce que le Code civil pose un critère fonctionnel (le but lucratif qui distingue l'activité commerciale des autres types d'activités), critère applicable aux personne morales dont le but est l'acroissement du profit, mais pas à leurs gérants, souvent subordonnés à un salaire. Et ce ne sont pas les personnes morales qui risquent d'encourir la détention provisoire...

Le législateur a essayé de réagir de manière casuistique. C'est la loi du 29 novembre 2012, également élaborée dans le cadre de la logique du Doing Business et retirant aux autorités répressives la compétence d'agir ex officio pour la poursuite de l'escroquerie, du détournement et de la dilapidation, de l'abus de confiance, commis en matière 
économique (dès lors uniquement sur la plainte de la victime, si l'acte concerné n'a pas causé de dommage à l'Etat), qui a trouvé une nouvelle formule. L'escroquerie et les autres infractions concernées sont considérées être commises en matière économique, lorsqu'il s'agit de l'acte d'un «commerçant individuel (artisan) dans le cadre de son activité professionnelle ou de la gestion de ses biens, utilisés pour son activité professionnelle, ou de l'acte d'un membre d'un ogane de direction d'une entreprise, lié à la réalisation des compétences relatives à la direction de l'entreprise ou lié à la réalisation par l'entreprise de son activité commerciale ou autre» (art. 20 du CPP russe). La Cour suprême fédérale russe a repris dans son arrêt d'assemblée plénière du 19 décembre 2013 exactement cette même formule pour préciser de nouveau le champ d'application de l'interdiction concernant la détention provisoire.

Est-ce la fin de la saga des précisions? II ne semble pas, car le médiateur en charge de la protection des droits des entrepreneurs auprès du Président russe a de nouveau annoncé il n'y a pas si longtemps (en avril 20 I 5) que le noveau chapitre du CPP relatif aux procédures dérogatoires en matière économique et financière, qu'il propose de faire adopter, doit contenir un nouveau critère, exhaustif et exact, permettant de déterminer tous les bénéficiaires de la logique du Doing Business en droit pénal. ${ }^{12}$ Peut-on déduire de cette initiative que toutes les tentatives législatives antérieures, celles de la loi du 7 avril 2010 et de la loi du 29 novembre 2012,ont jusqu'à présent échoué? Ou toutes ces expériences «alchimiques» en matière pénale sont tout simplement techniquement vouées à l'échec en raison du caractère impersonnel de l'économie moderne?

\subsection{La critique politique via l'égalité devant la justice}

La consécration en matière pénale de la logique du Doing Business a également provoqué la critique politique à travers le principe d'égalité devant la justice et le risque du retour aux structures sociales hiérarchisées relevant quasiment du Moyen Age. Comme la doctrine russe l'a noté, en faisant le choix de cette démarche conceptuelle, «le législater a renoncé à l'égalitarisme au profit de l'élitarisme»(ILIUTCHENKO, p. 68). En fait, comment justifier la différence de deux régimes juridiques fondée sur l'activité professionnelle, voire établissant un critère d'appartenance fondé sur la catégorie socioprofessionnelle (commerçant ou non commerçant, gérant de l'entreprise ou quelqu'un d'autre)?

C'est peut-être cette différenciation sociale, à vrai dire difficillement justifiable et acceptable dans une société moderne, qui a provoqué, après l'adoption des lois du 29 décembre 2009 et du 7 avril 2010, interdisant la détention provisoire en matière économique et financière, l'attitude expectative des juges qui n'ont pas voulu se jeter

${ }^{12}$ Cf., par exemple: http://rbcdaily.ru/economy/562949994968778 
corps et âmes dans l'application directe de la nouvelle loi, en refusant la détention sous le seul prétexte formel de l'activité commerciale du mis en examen, et qui ont commencé, dans certains cas, à chercher une argumentation plus sophistiquée pour contourner cette démarche législative. Dans cette perspective, la Chambre sociale de la Fédération de Russie ${ }^{13}$ s'est même spécialement réunie le 6 décembre 2010 pour constater que l'application de la nouvelle loi du 7 avril 2010 trouve des « difficultés évidentes, le texte de la loi étant parfois tout simplement ignoré par les autorités compétentes... ». ${ }^{14} E n$ septembre 2017 une nouvelle table ronde, organisée par cette Chambre sociale, a apporté les mêmes constatations. ${ }^{15}$

C'est la Cour constitutionnelle russe qui a finalement été confrontée à ce problème, saisie par le tribunal de la ville de Salekhard (dans le nord de la Russie), dans le cadre d'une procédure russe équivalente à la question prioritaire de constitutinnalité, pour reprendre cette notion française. A vrai dire, cette affaire concernait le droit pénal de fond, qui depuis la loi du 29 novembre 2012 suit également la logique du Doing Businesset reconnaît une différenciation entre l'escroquerie ordinaire (art. I 59 du CP) et l'escroquerie en matièreéconomique, liée à l'inexécution des obligations contractuelles, où la peine encourue est beaucoup moins importante (art 159.4 du CP). Mais il est clair que la question, posée par le tribunal de Salekhard, englobe conceptuellement toute la matière, voire la logique du Doing Business sur le plan pénal en tant que telle, y compris évidemment les procédures dérogatoires en matière économique et financière.

Dans sa requête constitutionnelle le tribunal de Salekhard, après avoir suspendu le procès contre une personne mise en examen pour une escroquerie relative à l'inéxecution frauduleuse de plusieurs contrats dans le cadre de son activité commerciale et ayant causé un dommage aux particuliers correspondant à environ 180000 euros, a mis en doute la constitutionnalité de ces dispositions pénales, quidevaient les conduire à appliquer une peine plus légère, que celle qu'ils devraient prononcer envers une autre personne accusée d'escroquerie dans le cadre ordinaire « non commercial ». Selon le tribunal de Salekhard, une telle démarche viole le principe constitutionnel d'égalité devant la loi.

Dans son arrêt du II décembre 2014 la Cour constitutionnelle a reconnu l'inconstutionnalité de l'art. I 59.4 du CP russe, sans pour autant complétement écraser la logique du Doing Business. Plus concrétement, selon la Cour constitutionnelle, le

\footnotetext{
${ }^{13}$ Il s'agit de l'organe officiel, composé de personnes partiellement envoyées par les entités de la Fédération, partiellement désignées par le pouvoir central, et enfin par la société civile elle-même, qui représente en Russie la société civile pour officiellement présenter ses vœux devant le pouvoir. ${ }^{14}$ Extraits du texte de la lettre non publiée de la Chambre sociale du 24 novembre 2010 invitant à la réunion spéciale consacrée aux problèmes de l'application de la loi du 7 avril 2010.

${ }^{15} \mathrm{Cf}$ : http://www.garant.ru/news// I36268/
} 
législateur peut différencier l'incrimination d'escroquerie, en créant notamment une incrimination sui generis en matière de droit des affaires pour mieux protéger les entrepreneurs, éviter l'instrumentalisation du droit pénal en vue du réglement des conflits commerciaux etc. C'est-à-dire, l'art. I59.4 du CP reste légitime. Cependant, la responsabilité pénale dans le cadre de l'escroquerie en matière économique ne peut pas être a priori moins importante que dans le cadre de l'escroquerie ordinaire. Elle doit être équilibrée et dépendre du dommage causé, nonobstant la qualification des faits selon l'art. I 59 ou l'art. I 59.4 du CP.La Cour constitutionnelle a fixé un délai de 6 mois pour modifier les dispositions concernées du CP. Ces modifications n'ont pas eu lieu.En l'absence d'intervention législative, le II juin 20 I 5 l'art. I 59.4 a perduautomatiquement sa force juridique.

Si la logique de la Cour constitutionnelle est assez claire, l'application de sa décision et l'équilibrage symétrique des art. I 59 et I 59.4 du CP concernantles peines auraient privé l'art. I 59.4 du CP de son intérêt, ce qui explique les hésitations du législateur. A quoi sert la réforme pénale du 29 novembre 2012 si la peine encourue pour escroquerie en matière économique devient la même que sous l'empire de l'incrimination générale ? C'est pour cette raison, semble-t-il, que les promoteurs de la logique du Doing Business parmi les députés ont tout de suite lancé certaines initiatives législatives, permettant de rééquilibrer partiellement les art. 159 et 159.4 du CP, sans pour autant abandonner le caractère favorable de l'art. 159.4 du CP (KORNIA, p. 2).Pourtant, même si ce compromis aurait permis de «sauver» l'art. I59.4 du CP, conceptuellement il ne s'agit que de tentatives pour contourner l'arrêt de la Cour constitutionnelle du I I décembre 2014 et non de réellement l'exécuter. II a fallu une année pour décider et réagir. La loi du 3 juillet 2016 a apporté des modifications, cette fois-ci à l'art. 159, en ajoutant un nouvel alinéa 5 relatif à «l'escroquerie liée à l'inexécution préméditée des obligations contractuelles en matière commerciale ». Vu que les peines pour cette escroquerie sont absolument identiques à celles concernant l'escroquerie de droit commun, peut-on dire que la décision constitutionnelle est respectée? Pas tout à fait, car si la loi du 3 juillet 2016 ne différencie pas les peines, elle différencie les montants du dommage pénalisant l'escroquerie : ils sont beaucoup plus importants lorsqu'il s'agit de l'activité commerciale, que dans les cas où il s'agit du droit commun. Autrement dit, un acte qualifié d'infraction pénale pour un particulier ne sera pas une infraction pénale pour un homme d'affaires, sauf si le montant du dommage atteint un certain seuil. Dans ce contexte, une question se pose : est-ce que l'arrêt de la Cour constitutionnelle russe du I I décembre 20 I 4 est exécuté ou est, en quelques sorte, contourné?

Et cette situation avec les articles 159 et 159.4 du CP souligne les problèmes fondamentaux relatifs à l'application de la logique du Doing Business en matière pénale, problèmes qui dépassent le cadre technique pour entrer dans le domaine politicoconstitutionnel, voire purement politique. 


\section{CONCLUSION}

Pourconclure, le législateur russe continue à rechercher cette formule magique et salvatricequi permettra de dissocier l'activité économique légitime de tout risque pénal. Même s'il est allé assez loin dans la consécration de la logique du Doing Business en matière pénale, les réformes n'ont pas porté leurs fruits et ont vite démontré toutes les faiblesses de cette logique, tant au niveau technique que politique. Est-ce un résultat conceptuellement prévisible ? II semblerait. Le rôle du droit pénal ne réside pas dans la libération du terrain économique, où la logique du Crime Control doit complétement céder la place à celle du Doing Business, mais plutôt en la délimitation des frontières entre un comportement autorisé et un comportement interdit, ce qui permet de distinguer entre une économie propre et une économie «sale». Et si le droit pénal est de fait absent dans la «zone propre», n'agissant que préventivement, il ne peut appliquer que la logique du Crime Control dans la «zone sale». De là résulte l'incompatibilité totale du droit pénal et de la logique du Doing Business. Mais cette incompatibilité ne réside pas en l'absence du droit pénal dans l'économie, le droit pénal veillant toujours à sa frontère, mais plutôt en l'absence du Doing Business dans le droit pénal. Et est-ce que ce n'est pas cette subtilité dans la compréhension qui manque à ceux qui cherchent en Russie ou ailleursla formule de la coexistence de l'économie et du droit pénal, une formule qui doit être plus classique que magique.

\section{RÉFÉRENCES}

BONFILS,Philippe. Droit pénal des affaires. Paris:Montchrestien. 2009.

D'HERVÉ, Nicolas. La magistrature face au management judiciaire. In: Revue de science criminelle et de droit pénal comparé, 2015, I.

GOLOVKO, Léonid. Panorama de droit pénal russe $(2008$ - 201 I). In: Revue pénitentiaire et de droit pénal, $201 \mathrm{I}, \mathrm{N}^{0} 4$.

ILIUTCHENKO, Natalia. Osvobojdenie ot ugolovnoi otvetstvennosti po delam o prestupleniakh $\vee$ sfere ekonomitcheskoi deiatelnosti: uslovia i mekhanism realizatsii. In: Predprinimatelskoie pravo, 2013. No3 (en russe).

Kontseptsiya modernizatsii ugolovnogo zakonodatelstva $\vee$ ekonomitcheskoi sfere. Moscou: Fond «Liberalnaya missiya». 2010 (en russe).

KORNIA Anastassia. Biznesmeny polutchat svoe.In: Vedomosti. 24 avril 20I5. 73 (3819) (en russe). 
Le Code pénal russe de 1997/trad. de L. Golovko, sous la dir. de J. Pradel. Paris: Cujas. 1998.

PACKER, Herbert. Two Models of the Criminal Process. In: University of Pennsylvania Law Review. 1964. Vol. I I3. No I.

SUTHERLAND, Edwin H. White Collar Criminality. In: American Sociological Review. 1940. Vol. 5. Nol.

Verkhovenstvo prava i problemy ego obespetchenia $\vee$ pravoprimenitelnoi praktike (mezhdunarodnaia kollektivnaya monografiya). Moscou: éd. Statut. 2009 (en russe). Version courte en anglais: Rule of Law in Russia - Issues of Implementation, Enforcement and Practice. Moscou: éd. Statut. 2009

Recebido em: 12/02/2019

Aprovado em: 28/04/2019 


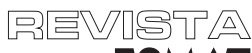

LÉONID GOLOVKO

ESMAT

252 REVISTA ESMAT

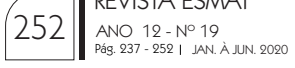

